\title{
Vaccinations in autoimmune inflammatory rheumatic diseases
}

\section{Leszek Szenborn}

Department of Pediatrics and Infectious Diseases of Medical University in Wroclaw, Poland

Pediatric patients with rheumatic diseases are at increased risk of infection due to the effect of immunosuppressive therapy and the disease itself. Current treatment strategies are based on the early use of immunosuppressive and biological drugs, which increases susceptibility to disease. Vaccination is a valuable method of preventing illness - from infectious diseases to autoimmune inflammatory rheumatic diseases (AIRD). However, the efficacy of vaccination in patients with AIRD can be reduced. The hypothetical risk of exacerbation of AIRD after vaccination was the cause of decades of under-performing vaccination in this group of patients. The issues related to the needs and safety of vaccination were discussed with Professor Lidia Rutkowska-Sak from the Clinic and Policlinic of Rheumatology of Developmental Age at the National Institute of Geriatrics, Rheumatology and Rehabilitation in Warsaw. Experts such as rheumatologists and vaccinologists participated in the discussion that took place during the conference of the Polish Society of Vaccinology entitled "Vaccinations in gastroenterology and rheumatology", held on March 19, 2016 in Warsaw.

For many years, thanks to the spectacular results of widespread immunization, it was possible to eliminate or reduce the incidence of most diseases that could be prevented by this method. Immunization has also been used for indirect protection of patients with AIRD, i.e. herd immunity and herd protection. In the last five years a fundamental change has been observed in the approach to the problem of the needs and safety of vaccination in patients with AIRDs. Recommendations of the European League Against Rheumatism (EULAR) published in 2011 [1] and disseminated from the outset in Polish language by the journal Medycyna Praktyczna [2] are the primary source of information in Europe.

The most current source of information (2015), not bringing any amendments to the EULAR recommendations, but significantly broadening the base of publica- tions on which these recommendations are based, is the practical elaboration by Groot et al. [3]. The original guidelines were based on the analysis of 131 items of literature and the experience of the authors (experts), voting using of Delphi method, and the overall compatibility of the working group members with regard to the recommendations was $91.7 \%$.

In the overall assessment, in a population of patients with AIRD, vaccines are safe and immunogenic. Methotrexate and glucocorticoids have no clearly adverse effect on the immunogenicity of vaccines. Treatment of biologics may accelerate the decrease in antibody levels over time, although the majority of patients during the first period after vaccination were appropriately secured.

Persistence of immune memory in children and adults with AIRD can be reduced, which indicates the need for booster doses of vaccination.

Publication of the recommendations of international organizations is a very important step in disseminating the methods of treatment and prevention. The prevalence of recommendations among physicians and patients and the use of the latest recommendations is another, perhaps even more difficult task than developing them. The importance of the issue is demonstrated by the research on this topic [4]. In the era of contesting vaccination as a method of prevention, even in healthy children, it can be a difficult task. However, the crisis of confidence in vaccination in the general population may lead to a situation in which patients with AIRD may be particularly harmed.

Vaccinations in patients with AIRD and evidence-based medicine (EBM). In several studies on vaccination in patients with AIRD clinically relevant endpoints are rated (e.g. illness, hospitalization, complications, death). The term "efficacy" of the vaccine is its ability to stimulate a protective immune response following vaccination.

\section{Address for correspondence}

Leszek Szenborn, Department of Pediatrics and Infectious Diseases, Medical University in Wroclaw, Chałubińskiego 2-2A,

50-368 Wrocław, Poland, e-mail: leszek.szenborn@umed.wroc.pl

Submitted: 21.11.2016; Accepted: 7.12.2016 
"Sero-protection" is measured by the percentage of vaccinated patients who have had the emergence or significant increase in the concentration of antibodies. The possibility to examine the immune response does not always correlate with the actual clinical effectiveness. EULAR recommends determining post-vaccination concentration of specific antibodies in patients receiving high doses of glucocorticoids ( $\geq 2 \mathrm{mg} / \mathrm{kg}$ or the total dose of $\geq 20 \mathrm{mg} /$ day for at least two weeks), or treated with rituximab; and their consideration in patients vaccinated during treatment with TNF antagonists. We have a well-defined correlation of protection (protection against illness/concentration of antibodies) after vaccination against tetanus (TT), diphtheria (D), hepatitis B, Haemophilus influenzae type b (Hib); to a lesser extent against measles, mumps and rubella (MMR), anti-pneumococcal vaccine, against poliomyelitis (IPV - inactivated poliomyelitis vaccine), varicella-zoster (VZV) and an unknown extent in the case of pertussis or meningococcal (very complicated study).

Recommended rules of conduct with a child suffering from AIRD:

1. In an early stage of the disease, an overview of the current status of immunization and illnesses is required. If there are losses or delays in the implementation of the vaccination program, their supplementation should be considered. Vaccination against varicella-zoster virus (VZV) is recommended before the start of immunosuppressive therapy. Possible adverse events and exacerbation of underlying disease that occurred after previous vaccination should be inquired about (relative contraindication to the administration of certain vaccines).

2. Vaccination should be performed in a stable period of the underlying disease.

The working group has established several recommendations and most important among them are:

1. When indicated inactivated vaccines can be administered to children treated with GKS, DMARDs and/or anti-TNF drugs.

2. It is recommended to perform pneumococcal and influenza vaccination, if possible, before the treatment with rituximab (anti-CD20).

3. In the case of indications for prevention of anti-tetanus patients treated with rituximab in the past six months, the administration of anti-tetanus immunoglobulin is suggested due to a possible reduction in immunogenicity of TT vaccination.

4. It is recommended to postpone vaccination with live attenuated vaccines (VZV, MMR) in children treated with high doses of DMARDs, GKS or biological agents. In individual cases, however, giving such a vaccine could be considered if the risk of infectious disease outweighs the risk associated with vaccination.

5. A booster dose of VZV and MMR vaccine may be considered for administration in patients receiving methotrexate (doses $<15 \mathrm{mg} / \mathrm{m}^{2} /$ week) or GKS in small doses $[5,6]$.

6. Vaccination is recommended for children under the preventive vaccination plan. Mandatory and recommended vaccines include immunization against tetanus, diphtheria, polio (IPV), Hib, hepatitis A, hepatitis $B$, pneumococcal vaccine, rabies, meningococcal disease and tick-borne encephalitis. At the time of publication of the EULAR recommendations [2] the meningococcal vaccine against serogroup $B$ (the dominant cause of meningococcal disease in Europe) was not yet registered. The safety and immunogenicity of the vaccine $4 C M e n B$ (Bexero) has been demonstrated in patients with asplenia and hypocomplementemia [7].

7. Annual influenza vaccination for all pediatric patients with rheumatic diseases should be considered, given that vaccinations are safe and immunogenic.

8. Vaccination against the HPV is recommended for girls in adolescence, especially in patients with systemic lupus erythematosus. The EULAR recommendation for caution because of the potential prothrombotic effect of HPV vaccine is no more valid. This unproven hypothesis was rejected on the basis of the studies which were not available when the publication was prepared [8].

Conclusions. Vaccination of children with autoimmune inflammatory diseases is needed. The calendar of vaccination requires an adjustment to the disease and the type and phase of vaccination. Vaccination of patients with rheumatic diseases may have reduced efficiency, but has a similar safety profile as in healthy patients.

The author has received support from Pfizer, Sanofi and Novartis to participate in scientific conferences and conducted educational activities for physicians commissioned by those entities.

\section{References}

1. Heijstek MW, Ott de Bruin LM, Bijl M, et al. EULAR recommendations for vaccination in paediatric patients with rheumatic diseases. Ann Rheum Dis 2011; 70: 1704-1712.

2. Szczepienie dzieci chorych na autoimmunologiczne choroby Zalecenia European League Against Rhematism (EULAR) http:// www.mp.pl/szczepienia/artykuly/wytyczne/71022,szczepienie-dzieci-chorych-na-autoimmunologiczne-choroby-reumatyczne-zalecenia-european-league-against-rheumatism-eular 
3. Groot N, Heijstek MW, Wulffraat NM. Vaccinations in paediatric rheumatology: an update on current developments. Curr Rheumatol Rep 2015; 17: 46.

4. Schipper K, Bakker M, De Wit M, et al. Strategies for disseminating recommendations or guidelines to patients: a systematic review. Implement Sci 2016; 11: 82.

5. Barbosa CM, Terreri MT, Rosário PO, et al. Immune response and tolerability of varicella vaccine in children and adolescents with systemic lupus erythematosus previously exposed to varicella-zoster virus. Clin Exp Rheumatol 2012; 30: 791-798.

6. Heijstek MW, Kamphuis S, Armbrust W, et al. Effects of the live attenuated measles-mumps-rubella booster vaccination on disease activity in patients with juvenile idiopathic arthritis: a randomized trial. JAMA 2013; 309: 2449-2456.

7. Martión-Torres F, Bernatowska E, Shcherbina A, et al. The immunogenicity of a serogroup $B$ meningococcal vaccine (4CMenB) in children with complement deficiency, asplenia or splenic dysfunction. Abstract book 20th International Pathogenic Neisseria Conference, 4th-9th September 2016. Manchester, United Kingdomhttp://www.ipnc2016.org/IPNC2016AbstractBook.pdf abstract 154, p. 244.

8. Naleway AL, Crane B, Smith N, et al. Absence of venous thromboembolism risk following quadrivalent human papillomavirus vaccination. Vaccine Safety Datalink, 2008-2011. Vaccine 2016; 34: $167-171$ 